\title{
Nonlocal semilinear evolution equations without strong compactness: theory and applications
}

\author{
Irene Benedetti ${ }^{1}$, Luisa Malaguti ${ }^{2}$ and Valentina Tadde ${ }^{3^{*}}$
}

\section{"Correspondence:}

valentina.taddei@unimore.it

${ }^{3}$ Dipartimento di Scienze Fisiche,

Informatiche e Matematiche,

Università di Modena e Reggio

Emilia, Modena, I-41125, Italy

Full list of author information is

available at the end of the article

\begin{abstract}
A semilinear multivalued evolution equation is considered in a reflexive Banach space. The nonlinear term has convex, closed, bounded values and a weakly sequentially closed graph when restricted to its second argument. No strong compactness is assumed, neither on the evolution operator generated by the linear part, or on the nonlinear term. A wide family of nonlocal associated boundary value problems is investigated by means of a fixed point technique. Applications are given to an optimal feedback control problem, to a nonlinear hyperbolic integro-differential equation arising in age-structure population models, and to a multipoint boundary value problem associated to a parabolic partial differential equation.

MSC: Primary 34G25; secondary 34B10; 34B15; 47H04; 28B20; 34H05
\end{abstract}

Keywords: nonlocal condition; semilinear evolution equation; fixed point theorems; optimal feedback control problem; diffusion problem; age-structure population model

\section{Introduction}

The paper deals with the following semilinear evolution inclusion in a reflexive Banach space $E$ :

$$
\left\{\begin{array}{l}
x^{\prime}(t) \in A(t) x(t)+F(t, x(t)), \quad \text { for a.a. } t \in[a, b], \\
L x=M(x),
\end{array}\right.
$$

where $\{A(t)\}_{t \in[a, b]}$ is a family of linear not necessarily bounded operators, $F:[a, b] \times E \multimap E$ is a multivalued map (multimap for short) and $L: C([a, b] ; E) \rightarrow E, M: C([a, b] ; E) \rightarrow E$ are a linear and a nonlinear operator respectively. See Section 3 for detailed assumptions.

The boundary condition considered is very general and includes the initial value problem, the two-point problem as well as several nonlocal conditions. For instance, the following two cases are covered by our general framework when $L x=x(a)$ :

(i) $M(y)=\frac{1}{b-a} \int_{a}^{b} g(y(t)) d t$ with $g: E \rightarrow E$ such that $\left\|g\left(x_{1}\right)-g\left(x_{2}\right)\right\| \leq \kappa\left\|x_{1}-x_{2}\right\|$ for every $x_{1}, x_{2} \in E$ and $\kappa D\left\|K^{-1}\right\|<1$;

(ii) $M(y)=\sum_{i=1}^{n} \alpha_{i} y\left(t_{i}\right)+y_{0}$, with $y_{0} \in E, \alpha_{i} \in \mathbb{R} \backslash\{0\}, t_{i} \in[a, b], i=1, \ldots, n$ and $D\left\|K^{-1}\right\| \sum_{i=1}^{n}\left|\alpha_{i}\right|<1$,

where $D$ is the uniform bound for the evolution operator $U$ generated by $\{A(t)\}_{t \in[a, b]}$ (see formula (2.1)) and $K$ is the invertible linear operator $L U(\cdot, a)$ (see hypothesis (L2)).

(c) 2013 Benedetti et al.; licensee Springer. This is an Open Access article distributed under the terms of the Creative Commons Attribution License (http://creativecommons.org/licenses/by/2.0), which permits unrestricted use, distribution, and reproduction in any medium, provided the original work is properly cited. 
Since the pioneering work of Byszewsky [1], nonlocal problems have been extensively studied due to being of interest in several contexts. For instance, the multipoint boundary value problem (case (ii) above) has better application in physics than the classical initial problem because it allows measurements at $t=t_{i} \in[a, b], i=1, \ldots, n$ rather than just at $t=0$. Abstract problems similar to (1.1) are considered, for example, in [2, 3] and [4] with the linear part generator of a $C_{0}$-semigroup or of a strongly continuous evolution operator; the semigroup and the evolution operator are always assumed to be compact there. In [5] a semilinear functional differential inclusion associated with a multivalued nonlocal boundary condition is considered, and in [6] an abstract problem as (1.1) is studied with a lower Scorza-Dragoni type nonlinearity; in both papers, regularity conditions by means of a measure of non-compactness on the nonlinearity are assumed. However, in [7] and [8] we proved the existence of classical solutions for the inclusion (1.1) associated with a two-point boundary condition by means of weak topology, thus avoiding hypotheses of compactness both on the semigroup generated by the linear part and on the nonlinear term $F$. The same is done in the present paper, for a wider class of boundary conditions, in order to find a result of existence for mild solutions of (1.1) (see Theorem 3.1). In particular, unlike [5] and [6], this allows to treat a class of nonlinear terms $F$ which are not necessarily compact-valued.

Problems with a nonlinear nonlocal boundary condition of type (1.1) occur in many fields like pharmacokinetics, neural networks, epidemiology models etc, see e.g. [9, Section 10.2]. In Section 4 we present several applications of the abstract result Theorem 3.1. More precisely, with our techniques we can handle diffusion problems of the form

$$
u_{t}=\Delta u+p\left(t, x, \int_{\Omega} u(t, \xi) d \xi\right) u(t, x)
$$

joined with several boundary conditions and, in example (4.1), associated with a feedback control. This model arises from population dynamics theory in the case of intraspecific competition for resources (see [10]), while we refer to [11] for recent achievements for its controllability.

Moreover, we can consider the following class of nonlinear hyperbolic integro-differential problems arising in population biology:

$$
\left\{\begin{array}{l}
u_{t}=-u_{a}-f\left(t, a, \int_{0}^{B} u(t, a) d a\right) u, \quad 0 \leq a \leq B, 0 \leq t \leq T, \\
u(0, a)=u_{0}(a), \quad 0 \leq a \leq B, \\
u(t, 0)=\int_{0}^{B} b(a) u(t, a) d a, \quad 0 \leq t \leq T .
\end{array}\right.
$$

They describe the time evolution of the age-structure of a population. The first models studying the age-structure of a population were the linear ones by McKendrick and Von Forster [12, 13]. The nonlinear model was introduced by Gurtin-MacCamy [14]. We are able to solve problem (1.2), writing it as an abstract problem of type (1.1). A similar approach for this type of models, but in a different setting, can be found in [15].

In Section 3 the abstract existence result is proved by means of a linearization argument and the application of the Ky Fan fixed point theorem. We investigate problem (1.1) in the space of continuous functions $C([a, b] ; E)$ endowed with the weak topology. As was done in [4], on the nonlinear term $F$ we assume a growth condition (see $\left.\left(\mathrm{F}^{\prime}\right)\right)$ stronger 
than the sublinear growth, but weaker than the global integrably boundedness; instead, the sublinear growth condition is enough for studying the Cauchy problem and we have, as a by-product, the weak compactness of the solution set (see [16] and also Remark 3.2).

In Section 2 some definitions and results used in the sequel of the paper are recalled.

As far as we know, all the results showed are new even for the case of a single-valued nonlinear term $F$.

\section{Preliminaries}

Let $(E,\|\cdot\|)$ be a reflexive Banach space, and let $E_{w}$ be the space $E$ endowed with the weak topology. We denote by $n B$ the closed ball centered at the origin and of radius $n$ of $E$, and for a set $A \subset E$, the symbol $\bar{A}^{w}$ denotes the weak closure of $A$. We take for granted that a bounded subset $A$ of a reflexive Banach space $E$ is weakly relatively compact. In the whole paper, we denote by $\|\cdot\|_{1}$ and $\|\cdot\|_{0}$ the $L^{1}([a, b] ; E)$-norm and the $C([a, b] ; E)$-norm respectively and by $v$ the Lebesgue measure on $[a, b]$. We recall (see [17, Theorem 4.3]) that a sequence $\left\{x_{n}\right\}$ weakly converges to an element $x \in C([a, b] ; E)$ if and only if

1. there exists $N>0$ such that for every $n \in \mathbb{N}$ and $t \in[a, b],\left\|x_{n}(t)\right\| \leq N$;

2. for every $t \in[a, b], x_{n}(t) \rightarrow x(t)$;

Denoting $\Delta=\{(t, s) \in[a, b] \times[a, b]: a \leq s \leq t \leq b\}$, we recall that a two-parameter family $\{U(t, s)\}_{(t, s) \in \Delta}$, where $U(t, s): E \rightarrow E$ is a bounded linear operator and $(t, s) \in \Delta$, is called an evolution system if the following conditions are satisfied:

1. $U(s, s)=I, a \leq s \leq b ; U(t, r) U(r, s)=U(t, s), a \leq s \leq r \leq t \leq b$;

2. $(t, s) \mapsto U(t, s)$ is strongly continuous on $\Delta$, i.e., the map $(t, s) \rightarrow U(t, s) x$ is continuous on $\Delta$ for every $x \in E$.

For every evolution system, we can consider the respective evolution operator $U: \Delta \rightarrow$ $\mathcal{L}(E)$, where $\mathcal{L}(E)$ is the space of all bounded linear operators in $E$.

We observe that since the evolution operator $U$ is strongly continuous on the compact set $\Delta$, by the uniform boundedness theorem, there exists a constant $D=D_{\Delta}>0$ such that

$$
\|U(t, s)\|_{\mathcal{L}(E)} \leq D, \quad(t, s) \in \Delta
$$

for details about evolution systems, we refer to $[18,19]$.

Let $G: L^{1}([a, b] ; E) \rightarrow C([a, b] ; E)$ be the Cauchy operator defined by

$$
G f(t)=\int_{a}^{t} U(t, s) f(s) d s \quad \text { for } t \in[a, b] .
$$

Finally, for the sake of completeness, we recall some well-known results that we will need in the main section.

Firstly, we state the Ky Fan fixed point theorem.

Theorem 2.1 ([20, Theorem 1]) Let $X$ be a Hausdorff locally convex topological vector space, let $V$ be a compact convex subset of $X$, and let $G: V \multimap V$ be an upper semicontinuous multimap with closed, convex values. Then $G$ has a fixed point.

Then we mention two results that are contained in the so-called Eberlein-Smulian theory. 
Theorem 2.2 ([21, Theorem 1, p.219]) Let $\Omega$ be a subset of a Banach space X. The following statements are equivalent:

1. $\Omega$ is relatively weakly compact;

2. $\Omega$ is relatively weakly sequentially compact.

Corollary 2.1 ([21, p.219]) Let $\Omega$ be a subset of a Banach space X. The following statements are equivalent:

1. $\Omega$ is weakly compact;

2. $\Omega$ is weakly sequentially compact.

We recall the Krein-Smulian theorem.

Theorem 2.3 ([22, p.434]) The convex hull of a weakly compact set in a Banach space $E$ is weakly compact.

In conclusion, we recall the Pettis measurability theorem, which we use in Section 4.

Theorem 2.4 ([23, p.278]) Let $(X, \Sigma)$ be a measure space, and let $E$ be a separable Banach space. Then $f: X \rightarrow E$ is measurable if and only iffor every $e \in E^{\prime}$ the function $e \circ f: X \rightarrow \mathbb{R}$ is measurable with respect to $\Sigma$ and the Borel $\sigma$-algebra in $\mathbb{R}$.

\section{Abstract result}

\subsection{Problem setting}

We consider the semilinear differential inclusion (1.1) under the following hypotheses:

(A) $\{A(t)\}_{t \in[a, b]}$ is a family of linear not necessarily bounded operators with $A(t): D(A) \subset E \rightarrow E, D(A)$ not depending on $t$ and dense in $E$, generating an evolution operator $U: \Delta \rightarrow \mathcal{L}(E)$;

(F1) for every $t \in[a, b]$ and $x \in E, F(t, x)$ is nonempty, convex and weakly compact, and for every $x \in E, F(\cdot, x):[a, b] \multimap E$ has a measurable selection;

(F2) for a.a. $t \in[a, b], F(t, \cdot): E_{w} \multimap E_{w}$ is weakly sequentially closed;

(L1) $L: C([a, b] ; E) \rightarrow E$ is a linear continuous operator;

(L2) the operator $K: E \rightarrow E$ defined as $K x=L U(\cdot, a) x$ is invertible;

(M) $M: C([a, b] ; E) \rightarrow E$ is a weakly sequentially continuous operator mapping bounded sets into bounded sets and such that

$$
\limsup _{\|u\|_{0} \rightarrow \infty} \frac{\|M(u)\|}{\|u\|_{0}}=l \quad \text { with } l<\frac{1}{D\left\|K^{-1}\right\|}
$$

We observe that if $L x=x(a)$, we have that $K$ equals the identity operator, thus condition (L2) is trivially satisfied. When $A(t) \equiv A$ is the abstract formulation of the Laplace operator, it generates a strongly continuous compact semigroup of contractions $S(t)$ such that $S(t) x=x$ if and only if $x=0$ (see, e.g., [4] and [24]). According to the Fredholm alternative, $S(t)-I$ is invertible for all $t \in(a, b]$, where $I$ denotes the identity operator. Hence, the associated periodic problem satisfies condition (L2).

In the remaining part of this section, we always assume the following condition on the multivalued map $F$. 
(F3) for each bounded subset $\Omega$ of $E$, there exists $\eta_{\Omega} \in L^{1}([a, b] ; \mathbb{R})$ such that, for a.a.

$$
t \in[a, b], \sup _{x \in \Omega}\|F(t, x)\| \leq \eta_{\Omega}(t) .
$$

Instead, for our main result (see Theorem 3.1), we need the stronger assumption as follows.

(F3') For every $n \in \mathbb{N}$, there exists $\varphi_{n} \in L^{1}([a, b] ; \mathbb{R})$ such that, for a.a. $t \in[a, b]$, $\sup _{\|x\| \leq n}\|F(t, x)\| \leq \varphi_{n}(t)$ and

$$
\liminf _{n \rightarrow \infty} \frac{1}{n} \int_{a}^{b} \varphi_{n}(s) d s=0
$$

The previous boundedness condition is anyway weaker than the one that is often used in literature in relation with boundary value problems, i.e., the following one.

$\left(\mathrm{F}^{\prime \prime}\right)$ There exists $\eta \in L^{1}([a, b] ; \mathbb{R})$ such that, for every $x \in E$ and a.a. $t \in[a, b],\|F(t, x)\| \leq$ $\eta(t)$.

We look for mild solutions, i.e., for functions that satisfy the following definition.

Definition 3.1 A continuous function $x:[a, b] \rightarrow E$ is said to be a mild solution of problem (1.1) if there exists a function $f \in L^{1}([a, b] ; E)$ such that $f(t) \in F(t, x(t))$ for a.a. $t \in[a, b]$ and

$$
\begin{aligned}
& x(t)=U(t, a) x(a)+\int_{a}^{t} U(t, s) f(s) d s, \quad \forall t \in[a, b], \\
& L x=M(x) .
\end{aligned}
$$

By the assumption of the invertibility of the operator $K$, it is possible to write the solution as

$$
x(t)=U(t, a) K^{-1}[M(x)-L G f]+\int_{a}^{t} U(t, s) f(s) d s, \quad \forall t \in[a, b] .
$$

Remark 3.1 If $E$ is a separable Banach space, according to the Kuratowski-Ryll-Nardzewski theorem (see [25, Theorem A]), the measurability of $F(\cdot, x)$ for every $x \in E$ is sufficient to obtain condition (F1). Moreover, in [25] sufficient conditions are given to obtain the existence of a strongly measurable selection for the multimap $F(\cdot, x)$ in not necessarily separable Banach spaces.

\subsection{Existence result}

Given $q \in C([a, b] ; E)$, let us denote

$$
S_{q}=\left\{f \in L^{1}([a, b] ; E): f(t) \in F(t, q(t)) \text { a.a. } t \in[a, b]\right\} .
$$

Note that with our hypotheses on $F$, the set $S_{q}$ is always nonempty as the following proposition shows.

Proposition 3.1 (see [16, Proposition 2.2]) Let $F:[a, b] \times E \multimap E$ be a multimap satisfying properties (F1), (F2) and (F3). Then, for every $q \in C([a, b] ; E)$, the set $S_{q}$ is nonempty. 
We denote by $T: C([a, b] ; E) \multimap C([a, b] ; E)$ the multioperator defined as

$$
\begin{aligned}
T(q)= & \left\{x \in C([a, b] ; E): x(t)=U(t, a) K^{-1}[M(q)-L G f]\right. \\
& \left.+\int_{a}^{t} U(t, s) f(s) d s, \forall t \in[a, b], f \in S_{q}\right\} .
\end{aligned}
$$

The fixed points of the multioperator $T$ are mild solutions of problem (1.1).

Fix $n \in \mathbb{N}$, let $Q_{n}$ be the closed ball centered at the origin and of radius $n$ of $C([a, b] ; E)$, and denote by $T_{n}=T \mathrm{~L}_{Q_{n}}: Q_{n} \multimap C([a, b] ; E)$ the restriction of the operator $T$ on the set $Q_{n}$. We show some properties of $T_{n}$.

Proposition 3.2 The multioperator $T_{n}$ has a weakly sequentially closed graph.

Proof Let $\left\{q_{m}\right\} \subset Q_{n}$ and $\left\{x_{m}\right\} \subset C([a, b] ; E)$ satisfying $x_{m} \in T_{n}\left(q_{m}\right)$ for all $m$ and $q_{m} \rightarrow q$, $x_{m} \rightarrow x$ in $C([a, b] ; E)$; we will prove that $x \in T_{n}(q)$.

Since $q_{m} \in Q_{n}$ for all $m$ and $q_{m}(t) \rightarrow q(t)$ for every $t \in[a, b]$, it follows that $\|q(t)\| \leq$ $\liminf _{m \rightarrow \infty}\left\|q_{m}(t)\right\| \leq n$ for all $t$. Moreover, $M$ is weakly sequentially continuous, and hence $M\left(q_{m}\right) \rightarrow M(q)$ in $E$. The fact that $x_{m} \in T_{n}\left(q_{m}\right)$ means that there exists a sequence $\left\{f_{m}\right\}, f_{m} \in S_{q_{m}}$, such that

$$
x_{m}(t)=U(t, a) K^{-1}\left[M\left(q_{m}\right)-L G f_{m}\right]+\int_{a}^{t} U(t, s) f_{m}(s) d s, \quad \forall t \in[a, b] .
$$

We observe that, according to (F3), $\left\|f_{m}(t)\right\| \leq \eta_{n B}(t)$ for a.a. $t$ and every $m$, i.e., $\left\{f_{m}\right\}$ is bounded and uniformly integrable and $\left\{f_{m}(t)\right\}$ is bounded in $E$ for a.a. $t \in[a, b]$. Hence, by the reflexivity of the space $E$ and by the Dunford-Pettis theorem (see [22, p.294]), we have the existence of a subsequence, denoted as the sequence, and a function $g$ such that $f_{m} \rightarrow g$ in $L^{1}([a, b] ; E)$.

Moreover, we have

$$
\int_{a}^{t} U(t, s) f_{m}(s) d s \rightarrow \int_{a}^{t} U(t, s) g(s) d s, \quad \forall t \in[a, b]
$$

Indeed, let $e^{\prime}: E \rightarrow \mathbb{R}$ be a linear continuous operator. By the linearity and continuity of the integral and the evolution operator $U(t, s)$, we have that the operator

$$
g \rightarrow e^{\prime}\left(\int_{a}^{t} U(t, s) g(s) d s\right)
$$

is a linear and continuous operator from $L^{1}([a, t] ; E)$ to $\mathbb{R}$ for all $t \in[a, b]$. Then, from the definition of the weak convergence, we have for every $t \in[a, b]$

$$
e^{\prime}\left(\int_{a}^{t} U(t, s) f_{m}(s) d s\right) \rightarrow e^{\prime}\left(\int_{a}^{t} U(t, s) g(s) d s\right)
$$

Moreover, $\left\|G f_{m}(t)\right\| \leq D\left\|\eta_{n B}\right\|_{1}$ for all $m$ and every $t \in[a, b]$. Consequently, $G f_{m} \rightarrow G g$ weakly in $C([a, b] ; E)$. Hence, for the linearity and continuity of the operator $L$, we have 
that $L G f_{m} \rightarrow L G g$. Finally, by the linearity and continuity of $K^{-1}$ (due to hypotheses (L1) and (L2)) and $U(t, s)$, we have that

$$
x_{m}(t) \rightarrow U(t, a) K^{-1}[M(q)-L G g]+\int_{a}^{t} U(t, s) g(s) d s=x_{0}(t), \quad \forall t \in[a, b]
$$

implying, for the uniqueness of the weak limit in $E$, that $x_{0}(t)=x(t)$ for all $t \in[a, b]$.

To conclude, we only have to prove that $g(t) \in F(t, q(t))$ for a.a. $t \in[a, b]$.

By Mazur's convexity theorem, we have the existence of a sequence

$$
\tilde{f}_{m}=\sum_{i=0}^{k_{m}} \lambda_{m i} f_{m+i}, \quad \lambda_{m i} \geq 0, \sum_{i=0}^{k_{m}} \lambda_{m i}=1
$$

satisfying $\tilde{f}_{m} \rightarrow g$ in $L^{1}([a, b] ; \mathbb{R})$ and, up to a subsequence, there is $N_{0} \subset[a, b]$ with Lebesgue measure zero such that $\tilde{f}_{m}(t) \rightarrow g(t)$ for all $t \in[a, b] \backslash N_{0}$. With no loss of generality, we can also assume that $F(t, \cdot): E_{w} \multimap E_{w}$ is weakly sequentially closed and $\sup _{\|x\| \leq n}\|F(t, x)\| \leq \eta_{n B}(t)$ for every $t \notin N_{0}$.

Fix $t_{0} \notin N_{0}$ and assume, by contradiction, that $g\left(t_{0}\right) \notin F\left(t_{0}, q\left(t_{0}\right)\right)$. By the reflexivity of the space $E$, the restriction $F_{n B}\left(t_{0}, \cdot\right)$ of the multimap $F\left(t_{0}, \cdot\right)$ on the set $n B$ is weakly compact. Hence, by Corollary $2.1, F_{n B}\left(t_{0}, \cdot\right)$ is a weakly closed multimap, and by [26, Theorem 1.1.5], it is weakly u.s.c. Since $\left\|q\left(t_{0}\right)\right\| \leq n$ and since $F_{n B}\left(t_{0}, q\left(t_{0}\right)\right)$ is closed and convex, from the Hahn-Banach theorem, there is a weakly open convex set $V \supset F_{n B}\left(t_{0}, q\left(t_{0}\right)\right)$ satisfying $q\left(t_{0}\right) \notin \bar{V}$. Since $F_{n B}\left(t_{0}, \cdot\right)$ is u.s.c., we can also find a weak neighborhood $V_{1}$ of $q\left(t_{0}\right)$ such that $F_{n B}\left(t_{0}, x\right) \subset V$ for all $x \in V_{1}$ with $\|x\| \leq n$. Note that $\left\|q_{m}\left(t_{0}\right)\right\| \leq n$ for all $m$. The convergence $q_{m}\left(t_{0}\right) \rightarrow q\left(t_{0}\right)$ as $m \rightarrow \infty$ then implies the existence of $m_{0} \in \mathbb{N}$ such that $q_{m}\left(t_{0}\right) \in V_{1}$ for all $m>m_{0}$. Therefore $f_{m}\left(t_{0}\right) \in F_{n B}\left(t_{0}, q_{m}\left(t_{0}\right)\right) \subset V$ for all $m>m_{0}$. The convexity of $V$ implies that $\tilde{f}_{m}\left(t_{0}\right) \in V$ for all $m>m_{0}$ and, by the convergence, we arrive at the contradictory conclusion that $g\left(t_{0}\right) \in \bar{V}$. We conclude that $g(t) \in F(t, q(t))$ for a.a. $t \in[a, b]$.

Proposition 3.3 The multioperator $T_{n}$ is weakly compact.

Proof We first prove that $T_{n}\left(Q_{n}\right)$ is weakly relatively sequentially compact.

Let $\left\{q_{m}\right\} \subset Q_{n}$ and $\left\{x_{m}\right\} \subset C([a, b] ; E)$ satisfying $x_{m} \in T_{n}\left(q_{m}\right)$ for all $m$. By the definition of the multioperator $T_{n}$, there exists a sequence $\left\{f_{m}\right\}, f_{m} \in S_{q_{m}}$, such that

$$
x_{m}(t)=U(t, a) K^{-1}\left[M\left(q_{m}\right)-L G f_{m}\right]+\int_{a}^{t} U(t, s) f_{m}(s) d s, \quad \forall t \in[a, b] .
$$

Moreover, reasoning as in Proposition 3.2, we have that there exist a subsequence, denoted as the sequence, and a function $g$ such that $f_{m} \rightarrow g$ in $L^{1}([a, b]$; $E)$. Since the operator $M$ maps bounded sets into bounded sets and $Q_{n}$ is bounded, we obtain that $M\left(q_{m}\right) \rightarrow \bar{x} \in E$ up to subsequence. Therefore

$$
x_{m}(t) \rightarrow l(t)=U(t, a) K^{-1}(\bar{x}-L G g)+\int_{a}^{t} U(t, s) g(s) d s, \quad \forall t \in[a, b] .
$$


Furthermore, by the weak convergence of $\left\{f_{m}\right\}$ and $\left\{M\left(q_{m}\right)\right\}$, we have

$$
\left\|x_{m}(t)\right\| \leq D\left\|K^{-1}\right\|\left[\left\|M\left(q_{m}\right)\right\|+\|L\| D\left\|f_{m}\right\|_{1}\right]+D\left\|f_{m}\right\|_{1} \leq N
$$

for all $m \in \mathbb{N}$, for all $t \in[a, b]$, and for some $N>0$. Reasoning again like in Proposition 3.2, it is then easy to prove that $x_{m} \rightarrow l$ in $C([a, b] ; E)$. Thus $T_{n}\left(Q_{n}\right)$ is weakly relatively sequentially compact, hence weakly relatively compact by Theorem 2.2 .

Proposition 3.4 The multioperator $T_{n}$ has convex and weakly compact values.

Proof Fix $q \in Q_{n}$, since $F$ is convex-valued, the set $T_{n}(q)$ is convex from the linearity of the integral and of the operators $K^{-1}, L$, and $U(t, s)$ for all $(t, s) \in \Delta$. The weak compactness of $T_{n}(q)$ follows by Propositions 3.3 and 3.2.

Theorem 3.1 Under the assumptions (A), (F1), (F2), (F3'), (L1), (L2) and (M), problem (1.1) has at least a mild solution.

Proof We show that there exists $n \in \mathbb{N}$ such that the operator $T_{n}$ maps the ball $Q_{n}$ into itself.

According to (3.2), there exists a subsequence, still denoted as the sequence, such that

$$
\lim _{n \rightarrow \infty} \frac{1}{n} \int_{a}^{b} \varphi_{n}(s) d s=0
$$

Assume by contradiction that there exist two sequences $\left\{q_{n}\right\}$ and $\left\{x_{n}\right\}$ such that $q_{n} \in Q_{n}$, $x_{n} \in T_{n}\left(q_{n}\right)$ and $x_{n} \notin Q_{n}$ for all $n \in \mathbb{N}$. By the definition of $T_{n}$, there exists a sequence $\left\{g_{n}\right\} \subset L^{1}([a, b] ; E), g_{n}(s) \in F\left(s, q_{n}(s)\right) \forall n \in \mathbb{N}$ and q.o. $s \in[a, b]$, such that

$$
x_{n}(t)=U(t, a) K^{-1}\left[M\left(q_{n}\right)-L G g_{n}\right]+\int_{a}^{t} U(t, s) g_{n}(s) d s, \quad \forall t \in[a, b] .
$$

By the assumption $x_{n} \notin Q_{n}$, we must have, for every $n$,

$$
n<\left\|x_{n}\right\|_{0} \leq D\left\|K^{-1}\right\|\left[\left\|M\left(q_{n}\right)\right\|+\|L\| D\left\|g_{n}\right\|_{1}\right]+D\left\|g_{n}\right\|_{1} .
$$

Moreover $q_{n} \in Q_{n}$ implies by $\left(\mathrm{F}^{\prime}\right)$ that $\left\|g_{n}(t)\right\| \leq \varphi_{n}(t)$ for a.a. $t \in[a, b]$, hence $\left\|g_{n}\right\|_{1} \leq$ $\left\|\varphi_{n}\right\|_{1}$. Consequently,

$$
n<D\left\|K^{-1}\right\|\left\|M\left(q_{n}\right)\right\|+D\left\|\varphi_{n}\right\|_{1}\left(\left\|K^{-1}\right\|\|L\| D+1\right) .
$$

Therefore

$$
\frac{1}{D\left\|K^{-1}\right\|}<\frac{\left\|M\left(q_{n}\right)\right\|}{n}+\frac{\left\|\varphi_{n}\right\|_{1}}{n}\left(\|L\| D+\frac{1}{\left\|K^{-1}\right\|}\right) .
$$

Notice that if $\left\|q_{n}\right\|_{0} \leq H<+\infty$ for every $n \in \mathbb{N}$, then $\lim _{n \rightarrow \infty} \frac{\left\|M\left(q_{n}\right)\right\|}{n}=0$ because $M$ maps bounded sets into bounded sets; if $\limsup _{n \rightarrow \infty}\left\|q_{n}\right\|_{0}=+\infty$, then by hypothesis we have

$$
\limsup _{n \rightarrow \infty} \frac{\left\|M\left(q_{n}\right)\right\|}{n} \leq \limsup _{n \rightarrow \infty} \frac{\left\|M\left(q_{n}\right)\right\|}{\left\|q_{n}\right\|_{0}} \leq \limsup _{\|u\| \rightarrow \infty} \frac{\|M(u)\|}{\|u\|_{0}}=l<\frac{1}{D\left\|K^{-1}\right\|}
$$


In both cases $\limsup _{n \rightarrow \infty} \frac{\left\|M\left(q_{n}\right)\right\|}{n}<\frac{1}{D\left\|K^{-1}\right\|}$. Moreover, by (3.4)

$$
\lim _{n \rightarrow \infty} \frac{\left\|\varphi_{n}\right\|_{1}}{n}\left(\|L\| D+\frac{1}{\left\|K^{-1}\right\|}\right)=0
$$

Hence

$$
\frac{1}{D\left\|K^{-1}\right\|} \leq \limsup _{n \rightarrow \infty}\left[\frac{\left\|M\left(q_{n}\right)\right\|}{n}+\frac{\left\|\varphi_{n}\right\|_{1}}{n}\left(\|L\| D+\frac{1}{\left\|K^{-1}\right\|}\right)\right]<\frac{1}{D\left\|K^{-1}\right\|}
$$

obtaining a contradiction.

Fix now $n \in \mathbb{N}$ such that $T_{n}\left(Q_{n}\right) \subseteq Q_{n}$. By Proposition 3.3 the set $V_{n}={\overline{T_{n}\left(Q_{n}\right.}}^{w}$ is a weakly compact set. Let now $W_{n}=\overline{\mathrm{co}}\left(V_{n}\right)$, where $\overline{\mathrm{co}}\left(V_{n}\right)$ denotes the closed convex hull of $V_{n}$. By Theorem 2.3, $W_{n}$ is a weakly compact set. Moreover, from the fact that $T_{n}\left(Q_{n}\right) \subset Q_{n}$ and that $Q_{n}$ is a convex closed set, we have that $W_{n} \subset Q_{n}$ and hence

$$
T_{n}\left(W_{n}\right)=T_{n}\left(\overline{\mathrm{co}}\left(T_{n}\left(Q_{n}\right)\right)\right) \subseteq T_{n}\left(Q_{n}\right) \subseteq{\overline{T_{n}\left(Q_{n}\right)}}^{w}=V_{n} \subset W_{n}
$$

Therefore, from Proposition 3.2 and from Corollary 2.1, we obtain that the restriction of the multimap $T_{n}$ on $W_{n}$ has a closed graph, and hence, by Proposition 3.4, it is weakly u.s.c (see [26, Theorem 1.1.5]). The conclusion then follows by Theorem 2.1.

When dealing with Cauchy problems, it is possible to weaken condition (F3') and to obtain the weak compactness of the solution set, as pointed out in the following remark.

Remark 3.2 In [27, Theorem 4.1], under condition (F3), an existence result for local classical solutions for the Cauchy problem associated to a semilinear differential inclusion is proved. Following the proof's outline of the cited theorem and combing it with Propositions 3.2, 3.3, 3.4, it is easy to obtain the existence of local mild solutions for (1.1) with a Cauchy initial condition under the same boundedness assumption (F3). Moreover, in the same paper (see Theorem 4.2), a global classical solution is obtained for the initial value problem under the following classical assumption.

$\left(\mathrm{F}^{\prime \prime \prime}\right)$ There exists $\alpha \in L^{1}([a, b] ; \mathbb{R})$ such that, for every $x \in E$ and a.a. $t \in[a, b],\|F(t, x)\| \leq$ $\alpha(t)(1+\|x\|)$.

Again, under (F3'"'), in [16, Theorem 4.2] the existence of a nonempty weakly compact set of mild solutions is showed for the initial value problem.

We point out the fact that the boundedness hypotheses considered are related as follows: condition (F3") implies (F3') which implies (F3"') which implies (F3). 


\section{Applications}

\subsection{Optimal feedback control problems of population diffusion models}

Let $\Omega$ be a bounded domain in $\mathbb{R}^{n}$ with boundary of class $C^{\infty}$ and $u:[0, d] \times \Omega \rightarrow \mathbb{R}$. We consider the following feedback control problem:

$$
\left\{\begin{array}{l}
u_{t}=\Delta u+p\left(t, x, \int_{\Omega} u(t, \xi) d \xi\right) u(t, x)+a(t, x) w(t, x)+b(t, x), \\
\quad t \in[0, d], x \in \Omega, w(t, x) \in W(u(t, x)), \\
u(t, x)=0, \quad t \in[0, d], x \in \partial \Omega, \\
u(0, x)=u_{0}(x), \quad x \in \Omega,
\end{array}\right.
$$

with $W(r)=\left\{s \in \mathbb{R}: \ell r+m_{1} \leq s \leq \ell r+m_{2}\right\}$, where $\ell>0$ and $m_{1}<m_{2}$.

We assume the following hypotheses:

(i) $a$ and $b$ are globally measurable in $[0, d] \times \Omega$, and there exist two functions $\varphi^{1}, \varphi^{2} \in L^{1}([0, d] ; \mathbb{R})$ such that, for a.a. $x \in \Omega$ and every $t \in[0, d]$,

$$
\begin{aligned}
& |a(t, x)| \leq \varphi^{1}(t) \\
& |b(t, x)| \leq \varphi^{2}(t)
\end{aligned}
$$

the map $p:[0, d] \times \Omega \times \mathbb{R} \rightarrow \mathbb{R}$ satisfies the following conditions:

(ii) for all $r \in \mathbb{R}, p(\cdot, \cdot, r):[0, d] \times \Omega \rightarrow \mathbb{R}$ is measurable;

(iii) for a.a. $t \in[0, d]$ and $x \in \Omega, p(t, x, \cdot): \mathbb{R} \rightarrow \mathbb{R}$ is continuous;

(iv) there exists $\varphi^{3} \in L^{1}([0, d] ; \mathbb{R})$ such that, for a.a. $x \in \Omega$ and every $t \in[0, d]$ and $r \in \mathbb{R}$,

$$
|p(t, x, r)| \leq \varphi^{3}(t)
$$

Let $y:[0, d] \rightarrow L^{2}(\Omega ; \mathbb{R}), v:[0, d] \rightarrow L^{2}(\Omega ; \mathbb{R}), f:[0, d] \times L^{2}(\Omega ; \mathbb{R}) \times L^{2}(\Omega ; \mathbb{R}) \rightarrow L^{2}(\Omega ; \mathbb{R})$, and $V: L^{2}(\Omega ; \mathbb{R}) \multimap L^{2}(\Omega ; \mathbb{R})$ be the maps defined by

$$
\begin{aligned}
& y(t)=u(t, \cdot) \\
& v(t)=w(t, \cdot) \\
& f(t, \alpha, \beta): \Omega \rightarrow \mathbb{R}, \quad f(t, \alpha, \beta)(x)=p\left(t, x, \int_{\Omega} \alpha(\xi) d \xi\right) \alpha(x)+a(t, x) \beta(x)+b(t, x) ; \\
& V(z)=\left\{v \in L^{2}(\Omega ; \mathbb{R}): \ell z(x)+m_{1} \leq v(x) \leq \ell z(x)+m_{2} \text {, a.a. } x \in \Omega\right\} .
\end{aligned}
$$

We can write the feedback control problem (4.1) as a first-order inclusion in the Hilbert space $E=L^{2}(\Omega ; \mathbb{R})$

$$
\left\{\begin{array}{l}
y^{\prime}(t) \in A y(t)+F(t, y(t)), \quad t \in[0, d], y(t) \in L^{2}(\Omega ; \mathbb{R}), \\
y(0)=y_{0},
\end{array}\right.
$$

where $F(t, z)=f(t, z, V(z)), A: W^{2,2}(\Omega ; \mathbb{R}) \cap W_{0}^{1,2}(\Omega ; \mathbb{R}) \rightarrow L^{2}(\Omega ; \mathbb{R})$ is the linear operator defined as $A y=\Delta y$ and $y_{0}=u_{0}(\cdot)$.

The operators $L: C([0, d] ; E) \rightarrow E$ and $M: C([0, d] ; E) \rightarrow E$ are defined as $L y=y(0)$ and $M(y)=y_{0}$. Trivially, $L$ is a linear and bounded operator, and $M$ is sequentially continuous 
with respect to the weak topology, and the operator $K$ of condition (L2) is the identity operator, in particular it is invertible. Moreover, it is known that $A$ generates a semigroup of contractions $U(t)$ on $E$ with the constant $D$ of (2.1) equal to 1 (see, e.g., [19] p.209-210). Hence hypotheses (A),(L1),(L2) and (M) are satisfied with $l=0$.

We prove that there exists a mild solution

$$
\begin{aligned}
& y(t)=U(t) y_{0}+\int_{0}^{t} U(t-s) f(s, y(s), v(s)) d s, \quad t \in[0, d], \\
& y(0)=y_{0},
\end{aligned}
$$

where $v(s) \in V(y(s)), s \in[0, d]$.

We show now that all the hypotheses of Theorem 3.1 are satisfied.

The map $g:[a, b] \rightarrow L^{2}(\Omega ; \mathbb{R})$ defined as

$$
g(t)(x)=p\left(t, x, \int_{\Omega} z(\xi) d \xi\right) z(x)+a(t, x)\left(\ell z(x)+m_{1}\right)+b(t, x)
$$

is a selection of $F(\cdot, z)$. Moreover, by the separability of the space $L^{2}(\Omega ; \mathbb{R})$, conditions (i), (ii), (iii), and by the Pettis measurability theorem (see Theorem 2.4), we obtain that $g$ is a measurable map, and so we have obtained for every $z \in L^{2}(\Omega ; \mathbb{R})$ the existence of a measurable selection of $F(\cdot, z)$.

We prove now that the map $F$ verifies condition (F2), i.e., that the map $F(t, \cdot)$ : $L^{2}(\Omega ; \mathbb{R}) \rightarrow L^{2}(\Omega ; \mathbb{R})$ is weakly sequentially closed for a.a. $t \in[0, d]$.

We start proving the sequential closedness with respect to the weak topology of the multimap $V$.

Let $\left\{\alpha_{j}\right\} \in L^{2}(\Omega ; \mathbb{R}), \alpha_{j} \rightarrow \alpha$ in $L^{2}(\Omega ; \mathbb{R}),\left\{\omega_{j}\right\} \in L^{2}(\Omega ; \mathbb{R}), \omega_{j} \in V\left(\alpha_{j}\right), \omega_{j} \rightarrow \omega$. According to Mazur's convexity lemma, for each $j$ there exist $k_{j} \in \mathbb{N}$ and positive numbers $\lambda_{j i}, i=0, \ldots, k_{j}$ such that $\sum_{i=0}^{k_{j}} \lambda_{j i}=1$ and the sequence $\tilde{\alpha}_{j}=\sum_{i=0}^{k_{j}} \lambda_{j i} \alpha_{j+i} \rightarrow \alpha$ in $L^{2}(\Omega ; \mathbb{R})$. Then we can extract a subsequence, denoted as the sequence, satisfying $\tilde{\alpha}_{j}(x) \rightarrow \alpha(x)$ for a.a. $x \in \Omega$. We have that the convex combination $\tilde{\omega}_{j}=\sum_{i=0}^{k_{j}} \lambda_{j i} \omega_{j+i}$ converges weakly to $\omega$. Moreover, by the definition of $V$, we have that

$$
\ell \tilde{\alpha}_{j}(x)+m_{1} \leq \tilde{\omega}_{j}(x) \leq \ell \tilde{\alpha}_{j}(x)+m_{2} \quad \text { a.a. } x \in \Omega
$$

Applying again Mazur's convexity lemma, for each $j$ there exist $h_{j} \in \mathbb{N}$ and positive numbers $v_{j i}, i=0, \ldots, h_{j}$ such that $\sum_{i=0}^{h_{j}} v_{j i}=1$ and the sequence $\tilde{\tilde{\omega}}_{j}=\sum_{i=0}^{h_{j}} v_{j i} \tilde{\omega}_{j+i} \rightarrow \omega$ in $L^{2}(\Omega ; \mathbb{R})$. Then we can extract a subsequence, denoted as the sequence, satisfying $\tilde{\tilde{\omega}}_{j}(x) \rightarrow$ $\omega(x)$ for a.a. $x \in \Omega$. As before,

$$
\ell \sum_{i=0}^{h_{j}} v_{j i} \tilde{\alpha}_{j+i}(x)+m_{1} \leq \sum_{i=0}^{h_{j}} v_{j i} \tilde{\omega}_{j+i}(x) \leq \ell \sum_{i=0}^{h_{j}} v_{j i} \tilde{\alpha}_{j+i}(x)+m_{2} .
$$

Then passing to the limit, we obtain

$$
\ell \alpha(x)+m_{1} \leq \omega(x) \leq \ell \alpha(x)+m_{2}, \quad \forall j, i=0, \ldots, h_{j}, \text { and a.a. } x \in \Omega,
$$

i.e., $\omega \in V(\alpha)$. 
Let now $t \in[0, d]$ be fixed, let $\left\{\alpha_{n}\right\} \subset L^{2}(\Omega ; \mathbb{R})$, weakly convergent to $\alpha \in L^{2}(\Omega ; \mathbb{R})$, and let $\left\{w_{n}\right\} \in L^{2}(\Omega ; \mathbb{R})$ with $w_{n} \in F\left(t, \alpha_{n}\right)$ for every $n \in \mathbb{N}$, weakly convergent to $w \in L^{2}(\Omega ; \mathbb{R})$. By the definition of the multimap $F$, we have

$$
w_{n}=f_{1}\left(t, \alpha_{n}\right)+f_{2}\left(t, \beta_{n}\right), \quad \text { with } \beta_{n} \in V\left(\alpha_{n}\right) \text { for every } n \in \mathbb{N},
$$

where $f_{1}, f_{2}:[0, d] \times L^{2}(\Omega ; \mathbb{R}) \rightarrow L^{2}(\Omega ; \mathbb{R}), f_{1}(t, \alpha)(x)=p\left(t, x, \int_{\Omega} \alpha(\xi) d \xi\right) \alpha(x), f_{2}(t, \beta)(x)=$ $a(t, x) \beta(x)+b(t, x)$.

By the definition of the multimap $V$ and the weak convergence of $\left\{\alpha_{n}\right\}$, we have that the sequence $\left\{\beta_{n}\right\}$ is norm bounded. Hence, by the reflexivity of the space $L^{2}(\Omega ; \mathbb{R})$, up to a subsequence, $\left\{\beta_{n}\right\}$ weakly converges to $\beta \in L^{2}(\Omega ; \mathbb{R})$ and the weak closure of the multimap $V$ implies $\beta \in V(\alpha)$. Moreover, by the continuity of the map $p$ and condition (iv), we have that $\left\{f_{1}\left(t, \alpha_{n}\right)\right\}$ converges weakly to $f_{1}(t, \alpha)$ and it is easy to see that $\left\{f_{2}\left(t, \beta_{n}\right)\right\}$ converges weakly to $f_{2}(t, \beta)$. In conclusion, we have obtained

$$
w=f_{1}(t, \alpha)+f_{2}(t, \beta) \in f(t, \alpha, V(\alpha))=F(t, \alpha) .
$$

Furthermore, easily, $V$ has convex and closed values; thus, by the linearity of the map $f_{2}$ and following the same reasonings as above, $F$ is convex closed-valued as well.

Finally, the multimap $F:[0, d] \times L^{2}(\Omega ; \mathbb{R}) \multimap L^{2}(\Omega ; \mathbb{R})$ verifies all the hypotheses of Theorem 3.1. Indeed from (i), (iv) and again from the definition of $V$, we have that

$$
\begin{aligned}
\|F(t, \alpha)\|_{2} & \leq\left\|f_{1}(t, \alpha)\right\|_{2}+\left\|f_{2}(t, V(\alpha))\right\|_{2} \\
& \leq \varphi^{3}(t)\|\alpha\|_{2}+\varphi^{1}(t)\left(\ell\|\alpha\|_{2}+m_{1}|\Omega|^{1 / 2}+\ell\|\alpha\|_{2}+m_{2}|\Omega|^{1 / 2}\right)+\varphi^{2}(t)|\Omega|^{1 / 2} \\
& \leq\left(\varphi^{3}(t)+2 \ell \varphi^{1}(t)\right)\|\alpha\|_{2}+|\Omega|^{1 / 2}\left[\left(m_{1}+m_{2}\right) \varphi^{1}(t)+\varphi^{2}(t)\right] .
\end{aligned}
$$

Denoting with $\varphi(t)=\varphi^{3}(t)+2 \ell \varphi^{1}(t)+|\Omega|^{1 / 2}\left[\left(m_{1}+m_{2}\right) \varphi^{1}(t)+\varphi^{2}(t)\right]$, we have

$$
\|F(t, \alpha)\|_{2} \leq \varphi(t)\left(1+\|\alpha\|_{2}\right)
$$

obtaining both that for every $t \in[0, d]$ and $\alpha \in L^{2}(\Omega ; \mathbb{R})$ the set $F(t, \alpha)$ is bounded (hence relatively compact by the reflexivity of $L^{2}(\Omega ; \mathbb{R})$ ) and that condition $\left(\mathrm{F} 3^{\prime \prime \prime}\right)$ is satisfied. Then applying Theorem 3.1 (see also Remark 3.2), we obtain the existence of a function such that

$$
y(t)=U(t) y_{0}+\int_{0}^{t} U(t-s) h(s) d s, \quad t \in[0, d]
$$

with $h(s) \in F(s, y(s))=f(s, y(s), V(y(s)))$.

Finally, applying the implicit function theorem of Filippov type (see [28, Theorem 7.2]), we have that there exists $v:[0, d] \rightarrow L^{2}(\Omega ; \mathbb{R})$ such that $v(t) \in V(y(t))$ and $h(t)=$ $f(t, y(t), v(t)), t \in[0, d]$.

Theorem 4.1 Let $j: C\left([0, d] ; L^{2}(\Omega ; \mathbb{R})\right) \rightarrow \mathbb{R}$ be a lower semicontinuous (l.s.c. for short) functional with respect to the weak topology. Then, under conditions (i)-(iv), there exists a 
mild solution $\left(u^{*}, \omega^{*}\right)$ of problem (4.1) such that

$$
j\left(u^{*}\right)=\min _{x \in \mathcal{S}\left(u_{0}\right)} j(x)
$$

where $\mathcal{S}\left(u_{0}\right)$ is the set of all mild trajectories of the system (4.1) with the initial value $u_{0}$.

Proof Under the hypotheses (i)-(iv), the set $\mathcal{S}\left(u_{0}\right) \neq \emptyset$; see the proof above. Moreover, according to [16, Theorem 4.2] (see also Remark 3.2), the solution set $\mathcal{S}\left(u_{0}\right)$ is weakly compact in $C\left([0, d], L^{2}(\Omega ; \mathbb{R})\right)$. Let $u^{*}$ be the minimizer of $j$ on $\mathcal{S}\left(u_{0}\right)$, and let $\omega^{*}$ be the corresponding control, then the pair $\left(u^{*}, \omega^{*}\right)$ is the required optimal solution.

\subsection{Age-structure population model}

We consider the nonlinear hyperbolic integro-differential problem (1.2) which was already introduced in Section 1. This model arises in population biology and describes the time evolution of the age-structure of a population. Here we consider the case of a nonlinear equation with linear boundary conditions. The independent variables $t$ and $a$ denote respectively time and age, and $u(t, a)$ represents the density of individuals of age $a$ at time $t$. The death rate $f$ is a nonnegative term depending on the time, the age and the total size of the population $\int_{0}^{B} u(t, a) d a$. The boundary condition accounts for the birth in the population. The weight function $b$ measures the fertility at age $a$.

We consider problem (1.2) under the following hypothesis:

(i) for all $c \in \mathbb{R}, f(\cdot, \cdot, c):[0, T] \times[0, B] \rightarrow \mathbb{R}$ is measurable;

(ii) for a.a. $t \in[0, T]$ and $a \in[0, B], f(t, a, \cdot): \mathbb{R} \rightarrow \mathbb{R}$ is continuous;

(iii) there exists $\alpha \in L^{1}([0, T] ; \mathbb{R})$ such that, for every $t \in[0, T], a \in[0, B]$ and $c \in \mathbb{R}$,

$$
|f(t, a, c)| \leq \alpha(t)
$$

(iv) $b \in L^{2}([0, B] ; \mathbb{R})$.

Problem (1.2) can be written as the following Cauchy problem in the Banach space $E=$ $L^{2}([0, B] ; \mathbb{R})$ :

$$
\left\{\begin{array}{l}
y^{\prime}(t)=A y(t)+F(t, y(t)) \\
y(0)=y_{0}
\end{array}\right.
$$

where $y:[0, T] \rightarrow E$ is defined as $y(t)=u(t, \cdot), y_{0}=u(\cdot), F:[0, T] \times E \rightarrow E$ is the singlevalued map $F(t, y)(a)=-f\left(t, a, \int_{0}^{B} y(s) d s\right) y(a)$ and $A: D(A) \rightarrow E$, with

$$
D(A)=\left\{y \in W^{1,2}([0, B] ; \mathbb{R}): y(0)=\int_{0}^{B} b(a) y(a) d a\right\},
$$

is the linear operator $A y=-y^{\prime} . A$ is the generator of the translation semigroup, which satisfies the identity

$$
U(t) y(a)= \begin{cases}y(a-t) & \text { if } t<a \\ \int_{0}^{B} b(s) U(t-a) y(s) d s & \text { if } t \geq a .\end{cases}
$$

This semigroup is intensely studied in $[29,30]$, thus we do not give any details here. We just recall that the translation semigroup is not compact. 
As showed in Section 4.1, the initial condition $y(0)=u_{0}$ satisfies (L1), (L2) and (M).

According to (iii), for every $y \in L^{2}([0, B] ; \mathbb{R})$, the function $a \rightarrow f\left(t, a \int_{0}^{B} y(s) d s\right) y(a)$ belongs to $L^{2}([0, B] ; \mathbb{R})$, hence $F$ is nonempty-valued. Moreover, the Pettis measurability theorem (see Theorem 2.4), the separability of $L^{2}([0, B] ; \mathbb{R}$ ) and conditions (i) and (ii) imply that $F$ is globally measurable (see [26, Corollary 1.3.1]), and hence, according to Remark 3.1, since $F$ is single-valued, condition (F1) is also satisfied.

We now prove that $F(t, \cdot)$ is weakly sequentially continuous for a.a. $t$, so we take $y_{n} \rightarrow y$ in $L^{2}([0, B], \mathbb{R})$. Then $\int_{0}^{B} y_{n}(s) d s \rightarrow \int_{0}^{B} y(s) d s$, thus (ii) implies that $f(t, a$, $\left.\int_{0}^{B} y_{n}(s) d s\right) \rightarrow f\left(t, a, \int_{0}^{B} y(s) d s\right)$ for a.a. $a \in[0, B]$. Moreover, it is possible to show that $f\left(t, a, \int_{0}^{B} y_{n}(s) d s\right) y_{n} \rightarrow f\left(t, a, \int_{0}^{B} y(s) d s\right) y$ in $L^{2}([0, B] ; \mathbb{R})$ obtaining condition (F2).

Finally, according to (iii), we have, for a.a. $t \in[0, T]$ and every $y \in L^{2}([0, B] ; \mathbb{R})$,

$$
\|F(t, y)\|_{2}^{2}=\int_{0}^{B} f^{2}\left(t, a, \int_{0}^{B} u(t, a) d a\right) y^{2}(a) d a \leq \alpha^{2}(t)\|y\|_{2}^{2},
$$

and so the growth condition (F3"') is satisfied. Recalling Remark 3.2, this condition is sufficient for the existence of at least a solution for the Cauchy problem.

Hence we find a mild solution $y \in C\left([0, T] ; L^{2}([0, B] ; \mathbb{R})\right)$. We stress that by (4.4) the solution $u(t, \cdot)=y(t)$ is a mild solution of (1.2) satisfying the required boundary conditions. Indeed,

$$
\begin{aligned}
u(t, 0) & =y(t)(0)=[U(0) y(t)](0)=\int_{0}^{B} b(s)[U(0) y(t)](s) d s=\int_{0}^{B} b(s) y(t)(s) d s \\
& =\int_{0}^{B} b(s) u(t, s) d s .
\end{aligned}
$$

Remark 4.1 With our techniques, we can attach also problems of the following general form:

$$
\left\{\begin{array}{l}
u_{t}=-u_{a}+\Delta u-f\left(t, a, x, \int_{0}^{B} u(t, a, x) d a\right) u, \quad 0 \leq a \leq B, 0 \leq t \leq T, x \in \Omega, \\
u(0, a, x)=u_{0}(a, x), \quad 0 \leq a \leq B, x \in \Omega, \\
u(t, a, x)=0, \quad 0 \leq a \leq B, 0 \leq t \leq T, x \in \partial \Omega, \\
u(t, 0, x)=\int_{0}^{B} b(a, x) u(t, a, x) d a, \quad 0 \leq t \leq T, x \in \Omega,
\end{array}\right.
$$

where the Laplacian operator is understood only with respect to the space variable $x$ and $\Omega \subset \mathbb{R}^{2}$ is a bounded closed domain with Lipschitz boundary. This model represents the age-structure of a population with spatial diffusion which takes into account the consequences of the environment changes on populations dynamics (see [31, 32]). The same arguments as above show that Theorem 3.1 applies to problem (4.5), taking again into account Remark 3.2. In fact, in this case, given the Banach space $E=L^{2}([0, B] \times \Omega ; \mathbb{R})$, we define the operator $A y=-\frac{\partial y}{\partial a}+\Delta y$ on

$$
D(A)=\left\{y: y(a, \cdot) \in W^{2,2}(\Omega ; \mathbb{R}), y(\cdot, x) \in W^{1,2}([0, B] ; \mathbb{R}), y(0, x)=\int_{0}^{B} b(a, x) y(a, x) d a\right\} .
$$

This operator generates a strongly continuous semigroup (see [33]), and all the other conditions can be shown as in the previous example. 


\subsection{Multipoint boundary value problem}

Let $t \in[a, b]$ and $\Omega \subset \mathbb{R}^{m}$ be a bounded set with a sufficiently regular boundary. Consider the multipoint boundary value problem

$$
\left\{\begin{array}{l}
u_{t} \in \Delta u+\left[p_{1}\left(t, x, \int_{\Omega} k(x, \xi) u(t, \xi) d \xi\right), p_{2}\left(t, x, \int_{\Omega} k(x, \xi) u(t, \xi) d \xi\right)\right] \varphi(t, x), \\
\quad t \in[a, b], x \in \Omega \\
u(t, x)=0, \quad t \in[a, b], x \in \partial \Omega, \\
u(a, x)+\sum_{i=1}^{n} c_{i} u\left(t_{i}, x\right)=u_{0}(x), \quad x \in \Omega
\end{array}\right.
$$

under the following hypotheses:

(i) $k: \Omega \times \Omega \rightarrow \mathbb{R}$ is measurable with $k(x, \cdot) \in L^{2}(\Omega ; \mathbb{R})$ and $\|k(x, \cdot)\|_{2} \leq 1$ for all $x \in \Omega$;

(ii) $p_{i}:[a, b] \times \Omega \times \mathbb{R} \rightarrow \mathbb{R}, i=1,2$, satisfy the following conditions:

(a) for every $r \in \mathbb{R}$ and $i=1,2, p_{i}(\cdot, \cdot, r)$ is measurable;

(b) for a.a. $t \in[a, b]$ and all $x \in \Omega, p_{1}(t, x, \cdot)$ is l.s.c. and $p_{2}(t, x, \cdot)$ is u.s.c.;

(c) there exist $\eta \in L^{1}([a, b] ; \mathbb{R})$ and $\lambda:[0, \infty) \rightarrow[0, \infty)$ increasing such that, for a.a. $t \in[a, b]$ and every $x \in \Omega, r \in \mathbb{R}$ and $i=1,2,\left|p_{i}(t, x, r)\right| \leq \eta(t) \lambda(|r|)$ and

$$
\liminf _{r \rightarrow \infty} \frac{\lambda(r)}{r}=0
$$

(d) for every $t \in[a, b], x \in \Omega$ and $r \in \mathbb{R}, p_{1}(t, x, r) \leq p_{2}(t, x, r)$;

(iii) $\varphi:[a, b] \times \Omega \rightarrow \mathbb{R}$ is measurable with $\varphi(t, \cdot) \in L^{2}(\Omega ; \mathbb{R})$ for a.a. $t \in[a, b]$ and $\|\varphi(t, \cdot)\|_{2} \in L^{\infty}([a, b] ; \mathbb{R}) ;$

(iv) $u_{0} \in L^{2}(\Omega ; \mathbb{R})$;

(v) $t_{i} \in[a, b], c_{i} \in \mathbb{R} \backslash\{0\}, i=1,2, \ldots, n$ satisfy $a<t_{1}<t_{2}<\cdots<t_{n} \leq b$ and $\sum_{i=1}^{n}\left|c_{i}\right|<1$. We search for solutions $u \in C\left([a, b] ; L^{2}(\Omega ; \mathbb{R})\right)$, and we can transform problem (4.6) into the nonlocal boundary value problem

$$
\left\{\begin{array}{l}
y^{\prime}(t) \in A y(t)+F(t, y(t)), \\
y(a)+\sum_{i=1}^{n} c_{1} y\left(t_{i}\right)=y_{0}
\end{array}\right.
$$

in the reflexive Hilbert space $L^{2}(\Omega ; \mathbb{R})$, where $y:[a, b] \rightarrow L^{2}(\Omega ; \mathbb{R}), A$ and $y_{0}$ are as in Section 4.1. For $\alpha \in L^{2}(\Omega ; \mathbb{R})$, the function $I_{\alpha}: \Omega \rightarrow \mathbb{R}$ defined by $I_{\alpha}(x)=\int_{\Omega} k(x, \xi) \alpha(\xi) d \xi$ is well defined and measurable according to (i). Moreover, $\left|I_{\alpha}(x)\right| \leq\|\alpha\|_{2}$ for every $x \in \Omega$. The multimap $F:[a, b] \times L^{2}(\Omega ; \mathbb{R}) \multimap L^{2}(\Omega ; \mathbb{R})$ is defined by $y \in F(t, \alpha)$ if and only if there is a measurable function $\beta: \Omega \rightarrow \mathbb{R}$ satisfying $p_{1}\left(t, x, I_{\alpha}(x)\right) \leq \beta(x) \leq p_{2}\left(t, x, I_{\alpha}(x)\right)$ and $y(x)=\beta(x) \varphi(t, x)$ for a.a. $x \in \Omega$. This definition is well posed, i.e., $F$ is nonempty-valued according to (ii) and (iii), and it is easy to see that $F$ is also convex-valued.

Given $(t, \alpha) \in[a, b] \times L^{2}(\Omega ; \mathbb{R})$, from (ii) we have that

$$
\begin{aligned}
\|y\|_{2} & =\sqrt{\int_{\Omega} \beta^{2}(x) \varphi^{2}(t, x) d x} \\
& \leq \sqrt{\int_{\Omega} \max \left\{p_{1}^{2}\left(t, x, I_{\alpha}(x)\right), p_{2}^{2}\left(t, x, I_{\alpha}(x)\right)\right\} \varphi^{2}(t, x) d x} \\
& \leq \eta(t) \lambda\left(\|\alpha\|_{2}\right)\|\varphi(t, \cdot)\|_{2},
\end{aligned}
$$


for every $y \in F(t, \alpha)$. Hence $F$ has weakly relatively compact values. Moreover, according to (4.9) and (ii)(c), we have that

$$
\sup _{\|\alpha\|_{2} \leq n}\|F(t, \alpha)\| \leq \eta(t) \lambda(n)\|\varphi(t, \cdot)\|_{2}:=\varphi_{n}(t) \quad \text { for } n \in \mathbb{N}
$$

and therefore (F3') follows from (4.7).

Now we investigate (F2), and hence we fix a value $t$ in $[a, b]$ for which (ii)(b) is satisfied and consider the sequences $\left\{\alpha_{n}\right\},\left\{y_{n}\right\} \subset L^{2}(\Omega ; \mathbb{R})$ such that $\alpha_{n} \rightarrow \alpha, y_{n} \rightarrow y$ in $L^{2}(\Omega ; \mathbb{R})$ and $y_{n} \in F\left(t, \alpha_{n}\right)$ for all $n \in \mathbb{N}$. Notice that the weak convergence of $\alpha_{n}$ implies both the existence of $\sigma>0$ such that $\left\|\alpha_{n}\right\|_{2} \leq \sigma$ and $I_{\alpha_{n}}(x) \rightarrow I_{\alpha}(x)$ for all $x \in \Omega$. Moreover, $y_{n}=\beta_{n} \varphi(t, \cdot)$ for all $n$ and some measurable $\beta_{n}: \Omega \rightarrow \mathbb{R}$ satisfying $p_{1}\left(t, x, I_{\alpha_{n}}(x)\right) \leq \beta_{n}(x) \leq p_{2}\left(t, x, I_{\alpha_{n}}(x)\right)$ for a.a. $x \in \Omega$. According to (ii)(c), $\left|\beta_{n}(x)\right| \leq \eta(t) \lambda(\sigma)$ a.e. in $\Omega$ for all $n$. Since $\Omega$ is bounded in $\mathbb{R}^{m}$, the set $\left\{\beta_{n}\right\} \subset L^{2}(\Omega ; \mathbb{R})$ and it is weakly relatively compact. Hence we can find a subsequence, again denoted as the sequence, satisfying $\beta_{n} \rightarrow \beta \in L^{2}(\Omega ; \mathbb{R})$. Consequently, applying Mazur's convexity lemma, for each $n$ there exist $k_{n} \in \mathbb{N}$ and positive numbers $\delta_{n i}, i=0,1, \ldots, k_{n}$, such that $\sum_{i=0}^{k_{n}} \delta_{n i}=1$ and the sequence $\tilde{\beta}_{n}:=\sum_{i=0}^{k_{n}} \delta_{n i} \beta_{n+i} \rightarrow \beta$. Up to a subsequence, denoted again as the sequence, $\tilde{\beta}_{n}(x) \rightarrow \beta(x)$ for a.a. $x \in \Omega$. Since

$$
\sum_{i=0}^{k_{n}} \delta_{n i} p_{1}\left(t, x, I_{\alpha_{n+i}}(x)\right) \leq \tilde{\beta}_{n}(x) \leq \sum_{i=0}^{k_{n}} \delta_{n i} p_{2}\left(t, x, I_{\alpha_{n+i}}(x)\right) \quad \text { for a.a. } x \in \Omega,
$$

passing to the limit as $n \rightarrow \infty$ and according to (ii)(b), we obtain that $p_{1}\left(t, x, I_{\alpha}(x)\right) \leq$ $\beta(x) \leq p_{2}\left(t, x, I_{\alpha}(x)\right)$ a.e. in $\Omega$. Let $\tilde{y}_{n}:=\sum_{i=0}^{k_{n}} \delta_{n i} y_{n+i}$. It is possible to see that $\tilde{y}_{n} \rightarrow y$. However, $\tilde{y}_{n}(x)=\tilde{\beta}_{n}(x) \varphi(t, x)$, and hence $\tilde{y}_{n}(x) \rightarrow \beta(x) \varphi(t, x)$ for a.a. $x \in \Omega$ and the convergence is dominated since $\left|\tilde{\beta}_{n}(x)\right| \leq \sum_{i=0}^{k_{n}} \delta_{n i}\left|\beta_{n}(x)\right| \leq \eta(t) \lambda(\sigma)$ for a.a. $x \in \Omega$. Therefore $\tilde{y}_{n} \rightarrow \beta \varphi(t, \cdot)$ in $L^{2}(\Omega ; \mathbb{R})$, and the uniqueness of the weak limit implies that $y=\beta \varphi(t, \cdot)$. We have showed that $F(t, \cdot)$ is weakly sequentially closed, i.e., that (F2) is satisfied, for a.a. $t \in[a, b]$. Then by (4.9) $F$ has weakly sequentially compact values since $L^{2}(\Omega ; \mathbb{R})$ is reflexive, and according to Corollary 2.1, it is weakly compact-valued. Furthermore, according to the Pettis measurability theorem (see Theorem 2.4), it is possible to show that the maps $t \mapsto p_{i}\left(t, \cdot, I_{\alpha}(\cdot)\right) \varphi(t, \cdot), i=1,2$, are measurable selection of $F(\cdot, \alpha)$ for every $\alpha \in L^{2}(\Omega ; \mathbb{R})$; hence condition (F1) is satisfied.

For $y \in L^{2}(\Omega ; \mathbb{R})$ we have that $L y=y(a)$ and then, as showed in Section 3, it satisfies both (L1) and (L2). Whereas $M: C\left([a, b] ; L^{2}(\Omega ; \mathbb{R})\right) \rightarrow L^{2}(\Omega ; \mathbb{R})$ is such that $M(y)=y_{0}-$ $\sum_{i=0}^{n} c_{i} y\left(t_{i}\right)$ and it satisfies condition (M) (see Introduction); estimate (3.1), in particular, depends on (v). All the assumptions of Theorem 3.1 are then satisfied, and hence problem (4.8) is solvable. It implies that the multipoint boundary value problem (4.6) has a solution $u \in C\left([a, b] ; L^{2}(\Omega ; \mathbb{R})\right)$.

Remark 4.2 Similarly as before, it is possible to show that also the nonlocal boundary value problem given by the differential inclusion in (4.6) associated with the boundary condition

$$
u(a, x)=\frac{1}{b-a} \int_{a}^{b} g(u(s, x)) d s, \quad x \in \Omega,
$$

is solvable, provided that $g: \mathbb{R} \rightarrow \mathbb{R}$ is Lipschitzian of some constant $k<1$. 
Remark 4.3 In (4.1) and (4.6) it is possible to substitute the Laplacian operator with a more general operator $L: W^{2,2}(\Omega ; \mathbb{R}) \cap W_{0}^{1,2}(\Omega ; \mathbb{R}) \rightarrow L^{2}(\Omega ; \mathbb{R})$ in divergence form of the following type:

$$
(L u)(x)=\sum_{i, j=1}^{n} \frac{\partial}{\partial x_{j}}\left(a_{i, j}(x) \frac{\partial}{\partial x_{i}} u(x)\right)
$$

with $t \in[a, b], x \in \Omega$ and

(a) $a_{i, j} \in L^{\infty}(\Omega), a_{i, j}=a_{j, i}, i, j=1,2, \ldots, n$;

(b) there exists $c>0$ such that, for a.a. $x \in \Omega$ and every $\xi \in \mathbb{R}^{n}, c\|\xi\|^{2} \leq \sum_{i, i=1}^{n} a_{i, j}(x) \xi_{i} \xi_{j}$.

\section{Competing interests}

The authors declare that they have no competing interests.

\section{Authors' contributions}

All authors have contributed equally. All authors read and approved the final manuscript.

\section{Author details}

${ }^{1}$ Dipartimento di Matematica e Informatica, Università di Perugia, Perugia, I-06123, Italy. ${ }^{2}$ Dipartimento di Scienze e Metodi dell'Ingegneria, Università di Modena e Reggio Emilia, Reggio Emilia, I-42122, Italy. ${ }^{3}$ Dipartimento di Scienze Fisiche, Informatiche e Matematiche, Università di Modena e Reggio Emilia, Modena, I-41125, Italy.

\section{Acknowledgements}

Dedicated to professor Jean Mawhin on the occasion of his seventieth birthday.

Supported by the national research project PRIN 2009 Ordinary Differential Equations and Applications.

Received: 20 November 2012 Accepted: 7 March 2013 Published: 26 March 2013

\section{References}

1. Byszewski, L: Theorems about the existence and uniqueness of a solutions of a semilinear evolution nonlocal Cauchy problem. J. Math. Anal. Appl. 162, 495-505 (1991)

2. Liu, Q, Yuanb, R: Existence of mild solutions for semilinear evolution equations with nonlocal initial conditions. Nonlinear Anal. 71, 4177-4184 (2009)

3. Paicu, A, Vrabie, II: A class of nonlinear evolution equations subjected to nonlocal initial conditions. Nonlinear Anal. 72, 4091-4100 (2010)

4. Papageorgiou, N: Existence of solutions for boundary value problems of semilinear evolution inclusions. Indian J. Pure Appl. Math. 23(7), 477-488 (1992)

5. Basova, MM, Obukhovskii, WV: On some boundary-value problems for functional-differential inclusions in Banach spaces. J. Math. Sci. 149(4), 1376-1384 (2008)

6. Cardinali, T, Portigiani, F, Rubbioni, P: Nonlocal Cauchy problems and their controllability for semilinear differential inclusions with lower Scorza-Dragoni nonlinearities. Czechoslov. Math. J. 61(136), 225-245 (2011)

7. Benedetti, I, Malaguti, L, Taddei, V: Two-points b.v.p. for multivalued equations with weakly regular r.h.s. Nonlinear Anal. 74, 3657-3670 (2011)

8. Benedetti, I, Malaguti, L, Taddei, V: B.V.P. for differential inclusions in Fréchet spaces with multiple solutions of the homogenous problem. Math. Bohem. 136(4), 367-375 (2011)

9. McKibben, MA: Discovering Evolution Equations with Applications, vol. I. Chapman \& Hall, Boca Raton (2011)

10. Gourley, SA: Travelling front solutions of a nonlocal Fisher equation. J. Math. Biol. 41, 272-284 (2000)

11. Allegretto, W, Nistri, P: Existence and optimal control for periodic parabolic equations with nonlocal terms. IMA J. Math. Control Inf. 16, 43-58 (1999)

12. McKendrick, AG: Applications of mathematics to medical problems. Proc. Edinb. Math. Soc. 44, 98-130 (1926)

13. Von Foerster, $\mathrm{H}$ : Some Remarks on Changing Populations, the Kinetics of Cellular Proliferation. Grune \& Stratton, New York (1959)

14. Gurtin, ME, MacCamy, RC: Non-linear age dependent population dynamics. Arch. Ration. Mech. Anal. 54, 281-300 (1974)

15. Benedetti, I, Taddei, V, Väth, M: Evolution problems with nonlinear nonlocal boundary conditions. J. Dyn. Diff. Eq. (to appear). doi:10.1007/s10884-013-9303-8

16. Benedetti, I, Malaguti, L, Taddei, V: Nonlocal semilinear evolution equations without strong compactness: theory and applications. Rend. Ist. Mat. Univ. Trieste 44, 371-388 (2012). (Special issue dedicated to the 60th birthday of Prof. F. Zanolin of Rend. Ist.)

17. Bochner, S, Taylor, AE: Linear functionals on certain spaces of abstractly-valued functions. Ann. Math. 39(4), 913-944 (1938)

18. Krein, SG: Linear Differential Equations in Banach Spaces. Am. Math. Soc., Providence (1971)

19. Pazy, A: Semigroups of Linear Operators and Applications to Partial Differential Equations. Springer, Berlin (1983) 
20. Fan, K: Fixed point and minimax theorems in locally convex topological linear spaces. Proc. Natl. Acad. Sci. USA 38 $121-126(1952)$

21. Kantorovich, LV, Akilov, GP: Functional Analysis. Pergamon, Oxford (1982)

22. Dunford, N, Schwartz, JT: Linear Operators. Wiley, New York (1988)

23. Pettis, BJ: On the integration in vector spaces. Trans. Am. Math. Soc. 44(2), 277-304 (1938)

24. Vrabie, II: Compactness Methods for Nonlinear Evolutions, 2nd edn. Longman, Harlow (1995)

25. Cascales, C, Kadets, V, Rodriguez, J: Measurability and selections of multi-functions in Banach spaces. J. Convex Anal. 17(1), 229-240 (2010)

26. Kamenskii, MI, Obukhovskii, W, Zecca, P: Condensing Multivalued Maps and Semilinear Differential Inclusions in Banach Space. de Gruyter, Berlin (2001)

27. Benedetti, I, Malaguti, L, Taddei, V: Semilinear differential inclusions via weak topologies. J. Math. Anal. Appl. 368 90-102 (2010)

28. Himmelberg, C: Measurable relations. Fundam. Math. 87, 53-72 (1975)

29. Alaoui, L: Age-dependent population dynamics and translation semigroups. Semigroup Forum 57(2), 186-207 (1998)

30. Grabosch, A: Translation semigroups and their linearizations on spaces of integrable functions. Trans. Am. Math. Soc. 311(1), 357-390 (1989)

31. Busenberg, S, lannelli, M: A degenerate nonlinear diffusion problem in age-structured population dynamics. Nonlinear Anal. 7(12), 1411-1429 (1983)

32. Liang, D, Sun, G, Wang, W: Second-order characteristic schemes in time and age for a nonlinear age-structured population model. J. Comput. Appl. Math. 235(13), 3841-3858 (2011)

33. Guo, BZ, Chan, WL: On the semigroup for age dependent population dynamics with spatial diffusion. J. Math. Anal. Appl. 184, 190-199 (1994)

doi:10.1186/1687-2770-2013-60

Cite this article as: Benedetti et al.: Nonlocal semilinear evolution equations without strong compactness: theory and applications. Boundary Value Problems 2013 2013:60.

\section{Submit your manuscript to a SpringerOpen ${ }^{\odot}$ journal and benefit from:}

- Convenient online submission

- Rigorous peer review

- Immediate publication on acceptance

- Open access: articles freely available online

- High visibility within the field

- Retaining the copyright to your article 